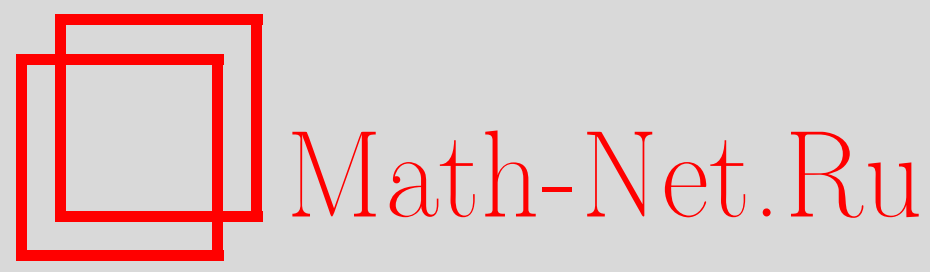

Г. М. Берколайко, Об одном функционале Петре, Матем. заметки, 1997, том 61, выпуск 1, 26-33

DOI: https://doi.org/10.4213/mzm1479

Использование Общероссийского математического портала Math-Net.Ru подразумевает, что вы прочитали и согласны с пользовательским соглашением http://www . mathnet.ru/rus/agreement

Параметры загрузки:

IP: 54.196.121.252

26 апреля 2023 г., 07:17:17

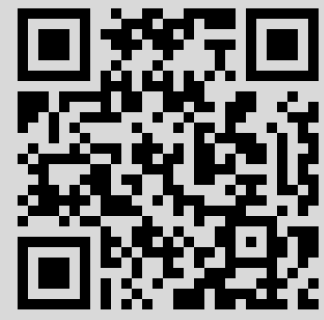




\section{ОБ ОДНОМ ФУНКЦИОНАЛЕ ПЕТРЕ}

\section{Г. М. Берколайко}

Для описания и изучения интерполяционных пространств вещественного метода обычно используется $K$-функционал Петре. В работе рассматривается его модификация $-K_{2}$-функционал Петре

$$
K_{2}(t, \mathbf{x})=\inf _{\mathbf{x}=\mathbf{x}_{1}+\mathbf{x}_{2}} \sqrt{\left\|\mathbf{x}_{1}\right\|_{1}^{2}+t^{2}\left\|\mathbf{x}_{2}\right\|_{2}^{2}}
$$

как функция от $t$ при фиксированном $\mathbf{x}$, выявляются его свойства, проводится рассмотрение некоторых частных случаев, рассматриваются классы функций, имеющих представление в виде $K_{2}(t)$.

Библиографоял: 3 названия.

Введение. Для описания и изучения интерполящионных пространств вещественного метода обычно используется $K$-функционал Петре

$$
K(t, \mathbf{x})=\inf _{\mathbf{x}=\mathbf{x}_{1}+\mathbf{x}_{2}}\left\|\mathbf{x}_{1}\right\|_{1}+t\left\|\mathbf{x}_{2}\right\|_{2}
$$

(см. [1]). Однако, уже сравнительно давно стало ясно, что при рассмотрении конкретных пар удобнее использовать его модификации. Так, в частности, для гильбертовых пар естественно использовать $K_{2}$-функционал Петре

$$
K_{2}(t, \mathbf{x})=\inf _{\mathbf{x}=\mathbf{x}_{1}+\mathbf{x}_{2}} \sqrt{\left\|\mathbf{x}_{1}\right\|_{1}^{2}+t^{2}\left\|\mathbf{x}_{2}\right\|_{2}^{2}}
$$

который при фиксированном значении параметра $t$ дает гильбертову норму. Более того, в случае гильбертовых пар оказьвается, что $K_{2}(t, \mathbf{x})$ как функция от $t$ обладает замечательным свойством. Он является лёвнеровской функцией (см. [2]). В данной работе делается попытка выявить дополнительные свойства, которьми обладает функция $K_{2}(t, \mathbf{x})=K_{2}(t)$ как функция от $t$ для произвольных банаховых пар.

В первом пункте доказывается непрерывная дифференцируемость функции $K_{2}(t)$, что отличает ее от функции $K(t)$. Во второй и третьей частях рассматриваются частные случаи в конечномерном (нормы - максимум координат и максимум с весом) и бесконечномерном $\left(\ell_{\infty}\right.$ и $\ell_{\infty}$ с весом $)$ пространствах. В четвертой части приводятся теоремы о функциях, имеющих представление в виде $K_{2}$-функционала. 
1. Основные свойства. Пусть $\left\{X_{1}, X_{2}\right\}$ - банахова пара. Функционал

$$
K_{2}(t, \mathbf{x})=\inf _{\mathbf{x}=\mathbf{x}_{1}+\mathbf{x}_{2}} \sqrt{\left\|\mathbf{x}_{1}\right\|_{1}^{2}+t^{2}\left\|\mathbf{x}_{2}\right\|_{2}^{2}}
$$

где $\mathbf{x}_{1} \in X_{1}, \mathbf{x}_{2} \in X_{2}, \mathbf{x} \in X_{1}+X_{2}$, рассматривается как функция от $t$ при фиксированном $\mathbf{X}$.

В дальнейшем нам понадобится известная лемма о сопряженной форме $K_{2}$-функционала (см. [1]).

ЛЕМмА 1. К

$$
K_{2}(t, \mathbf{x})=\sup _{f \neq 0} \frac{|f(\mathbf{x})|}{\left(\|f\|_{1}^{2}+t^{-2}\|f\|_{2}^{2}\right)^{1 / 2}}
$$

где $f(\mathbf{x})$ - линейньй функционал на $X_{1}+X_{2}$.

С помощью сопряженной формы мы, в частности, докажем следующую теорему.

ТеОРема 1. Функиия $K_{2}(t)$ непрерывно дифференцируема на $(0,+\infty)$.

ДокАЗАтельство. Рассмотрим $K^{*}(t)=\left(K_{2}(\sqrt{t})\right)^{2}$. Тогда

$$
K^{*}(t)=\inf _{\mathbf{x}=\mathbf{x}_{1}+\mathbf{x}_{2}}\left\|\mathbf{x}_{1}\right\|_{1}^{2}+t\left\|\mathbf{x}_{2}\right\|_{2}^{2}=\sup _{f \neq 0} \frac{|f(\mathbf{x})|^{2}}{\|f\|_{1}^{2}+t^{-1}\|f\|_{2}^{2}} .
$$

Как инфимум линейньх функций $K^{*}(t)$ - вогнутая функция, следовательно, из нарушений дифференцируемости могут быть только изломы (см. $[3,1.4 .3])$. Пусть $K^{*}(t)$ терпит излом в точке $t_{0}$. Тогда сушествует набор касательных $\varphi(t)=a t+b$ к графику функции $K^{*}(t)$ в точке $t_{0}$. Очевидно, что касательных больше, чем одна, и все они лежат над графиком $K^{*}(t)$. Рассмотрим преобразование $\bar{f}(t)=\left(f\left(t^{-1}\right)\right)^{-1}$. Тогда

$$
\overline{K^{*}}(t)=\inf _{f \neq 0} \frac{\|f\|_{1}^{2}}{|f(\mathbf{x})|^{2}}+t \frac{\|f\|_{2}^{2}}{|f(\mathbf{x})|^{2}}=\sup _{\mathbf{x}=\mathbf{x}_{1}+\mathbf{x}_{2}}\left(\left\|\mathbf{x}_{1}\right\|_{1}^{2}+t^{-1}\left\|\mathbf{x}_{2}\right\|_{2}^{2}\right)^{-1}
$$

Как инфимум линейных функций $\overline{K^{*}}(t)$ - вогнутая функция. Существует семейство касательных $\psi(t)=c t+d$ к графику $\overline{K^{*}}(t)$ в точке $t_{0}$. Функции $\varphi(t)$ перешли в непрерьвно дифференцируемые функции вида

$$
\bar{\varphi}(t)=\frac{t}{a+b t}
$$

которые касаются графика $\overline{K^{*}}(t)$ и лежат подним. Тогда будет вьполняться $\psi(t) \geqslant \bar{\varphi}(t)$ и $\psi\left(t_{0}\right)=\bar{\varphi}\left(t_{0}\right)$ для любых $\psi(t)$ и $\bar{\varphi}(t)$, т.е. любая $\psi(t)$ будет касательной для любой $\bar{\varphi}(t)$, чего не может быть. Следовательно, у $K^{*}(t)$ нет изломов. Применяя обратное преобразование $K_{2}(t)=\sqrt{K\left(t^{2}\right)}$, получаем, что $K_{2}(t)$ непрерьвно дифференцируема по $t$. 
2. Частный случай в конечномерном пространстве. Рассмотрим нормы на пространстве $\mathbb{R}^{n}$

$$
\begin{aligned}
& \|\mathbf{x}\|_{1}=\max \left(\alpha_{1}\left|x^{1}\right|, \alpha_{2}\left|x^{2}\right|, \ldots, \alpha_{n}\left|x^{n}\right|\right), \\
& \|\mathbf{x}\|_{2}=\max \left(\left|x^{1}\right|,\left|x^{2}\right|, \ldots,\left|x^{n}\right|\right) .
\end{aligned}
$$

Очевидно, что $K_{2}(t, \mathbf{x})=K_{2}(t,|\mathbf{x}|)$, где $|\mathbf{x}|=\left(\left|x^{1}\right|, \ldots,\left|x^{n}\right|\right)$. Поэтому при вычислении $K_{2}(t, \mathbf{x})$ мы будем без потери обшности предполагать, что $\mathbf{x}>0$ (т.е. $x^{i}>0$ ).

Лемма 2. Пусть $\mathbf{x}>0$. Тогда существует такое $h>0$, что инфимум в (1) достигается при разбиении $\mathbf{x}=\mathbf{y}_{1}+\mathbf{y}_{2}$ на векторы $\mathbf{y}_{1}, \mathbf{y}_{2}$ вида

$$
y_{1}^{i}=\left\{\begin{array}{ll}
x^{i}-h, & x^{i} \geqslant h, \\
0, & x^{i}<h ;
\end{array} \quad y_{2}^{i}= \begin{cases}h, & x^{i} \geqslant h, \\
x^{i}, & x^{i}<h .\end{cases}\right.
$$

ДоКАЗАТЕЛЬСТВо. В силу конечномерности инфимум достигается на некотором разбиении $\mathbf{x}=\mathbf{x}_{1}+\mathbf{x}_{2}$. Нетрудно видеть, что можно считать $\mathbf{x}_{1} \geqslant 0, \mathbf{x}_{2} \geqslant 0$. Тогда возьмем $h=\max _{i}\left(x_{2}^{i}\right)$ и построим соответствующее разбиение $\mathbf{x}=\mathbf{y}_{1}+\mathbf{y}_{2}$. Очевидно, $\left\|\mathbf{y}_{2}\right\|_{2}=h=\max _{i}\left(x_{2}^{i}\right)=\left\|\mathbf{x}_{2}\right\|_{2}$ и $\left\|\mathbf{y}_{1}\right\|_{1} \leqslant\left\|\mathbf{x}_{1}\right\|_{1}$, так как $y_{1}^{i} \leqslant x_{1}^{i}$. Следовательно, $\sqrt{\left\|\mathbf{y}_{1}\right\|_{1}^{2}+t_{0}^{2}\left\|\mathbf{y}_{2}\right\|_{2}^{2}} \leqslant \sqrt{\left\|\mathbf{x}_{1}\right\|_{1}^{2}+t_{0}^{2}\left\|\mathbf{x}_{2}\right\|_{2}^{2}}$. Лемма доказана.

Из леммы вытекает, что оптимальное разбиение следует искать среди разбиений вида (2).

При $n=1$ функция $K_{2}(t)$ преобразуется к виду

$$
K_{2}(t)=\inf _{h} \sqrt{\left(\alpha_{1}\left(x^{1}-h\right)\right)^{2}+t^{2} h^{2}} .
$$

Инфимум в (3) достигается при $h=\alpha_{1}^{2} x^{1} /\left(\alpha_{1}^{2}+t^{2}\right)$ или, что то же самое,

$$
t^{2}=\frac{\alpha_{1}^{2}\left(x^{1}-h\right)}{h} \text {. }
$$

Поэтому

$$
K_{2}(t)=x^{1} \sqrt{\frac{\alpha_{1}^{2} t^{2}}{\alpha_{1}^{2}+t^{2}}} .
$$

Пусть теперь $n \geqslant 1$. Обозначим

$$
f_{k}(t)=\inf _{h} \sqrt{\alpha_{k}^{2}\left(x^{k}-h\right)+t^{2} h^{2}}=x \sqrt{\frac{\alpha_{k}^{2} t^{2}}{\alpha_{k}^{2}+t^{2}}},
$$

при $1 \leqslant k \leqslant n$.

Очевидно, что $K_{2}(t) \geqslant f_{k}(t)$. Поэтому

$$
K_{2}(t) \geqslant \max _{1 \leqslant k \leqslant n} f_{k}(t) .
$$


ЛЕмма 3. Пусть гипербола $\psi(t)=\sqrt{a^{2}+b^{2} t^{2}}$ касается $f(t)=\max _{1 \leqslant k \leqslant n} f_{k}(t) u$ $f(t) \leqslant \psi(t)$ для любого $t$. Тогда существуют векторы $\mathbf{y}_{1}, \mathbf{y}_{2}$ такие, что $\mathbf{x}=\mathbf{y}_{1}+\mathbf{y}_{2}$, $\left\|\mathbf{y}_{1}\right\|_{1}=a,\left\|\mathbf{y}_{2}\right\|_{2}=b$.

ДокАЗАТЕльство. Так как $\psi(t)=\sqrt{a^{2}+b^{2} t^{2}}$ касается $f(t)$, то она должна касаться одной из $f_{k}(t)$. Пусть она касается $f_{i}(t)$ в точке $t_{i}$. Тогда существует такое $h_{i}$, что

$$
f_{i}\left(t_{i}\right)=\sqrt{\alpha_{i}^{2}\left(x^{i}-h_{i}\right)^{2}+h_{i}^{2} t_{i}^{2}}
$$

Обозначим

$$
\varphi(t)=\sqrt{\alpha_{i}^{2}\left(x^{i}-h_{i}\right)^{2}+h_{i}^{2} t^{2}}
$$

$\Phi$ ункция $\varphi(t)$ тоже касается $f_{i}(t)$ в точке $t_{i}$, так как $\varphi\left(t_{i}\right)=f_{i}\left(t_{i}\right)$ и

$$
f_{i}(t)=\inf _{h} \sqrt{\alpha_{i}^{2}\left(x^{i}-h_{i}\right)^{2}+h_{i}^{2} t_{i}^{2}} \leqslant \varphi(t)
$$

Отсюда вытекает, что $\psi\left(t_{i}\right)=f_{i}\left(t_{i}\right)=\varphi\left(t_{i}\right)$ и $\psi^{\prime}\left(t_{i}\right)=f_{i}^{\prime}\left(t_{i}\right)=\varphi^{\prime}\left(t_{i}\right)$. Поэтому гиперболы $\psi(t)$ и $\varphi(t)$ совпадут, т.е. $a=\alpha_{i}\left(x^{i}-h_{i}\right), b=h_{i}$.

Рассмотрим разбиение $\mathbf{x}=\mathbf{y}_{1}+\mathbf{y}_{2}$ вида $(2) \mathrm{c} h=h_{i}$. Докажем, что $\left\|\mathbf{y}_{1}\right\|_{1}=\alpha_{i}\left(x^{i}-h_{i}\right)$.

Предположим противное, т.е. что норма $\left\|\mathbf{y}_{1}\right\|_{1}$ вычисляется по другой координате: $\left\|\mathbf{y}_{1}\right\|_{1}=\alpha_{j}\left(x^{j}-h_{i}\right)$. Тогда $\alpha_{j}\left(x^{j}-h_{i}\right)>\alpha_{i}\left(x^{i}-h_{i}\right)$. Рассмотрим

$$
f_{j}(t)=\inf _{h} \sqrt{\alpha_{j}^{2}\left(x^{j}-h\right)^{2}+h^{2} t^{2}}
$$

Из формулы (4) определяется такое $t_{j}$, что инфимум в $(5)$ достигается при $h=h_{i}$, т.е. $f_{j}\left(t_{j}\right)=\sqrt{\alpha_{j}^{2}\left(x^{j}-h_{i}\right)^{2}+h_{i}^{2} t_{j}^{2}}$. Поэтому

$$
\begin{aligned}
\psi\left(t_{j}\right)=\varphi\left(t_{j}\right) & =\sqrt{\alpha_{i}^{2}\left(x^{i}-h_{i}\right)^{2} h_{i}^{2} t_{j}^{2}} \\
& <\sqrt{\alpha_{j}^{2}\left(x^{j}-h_{i}\right)^{2}+h_{i}^{2} t_{j}^{2}}=f_{j}\left(t_{j}\right) \leqslant f\left(t_{j}\right) .
\end{aligned}
$$

Получили противоречие: $\psi\left(t_{j}\right)<f\left(t_{j}\right)$. Лемма доказана.

Пусть $f$ - произвольная положительная функция, определенная на положительной полуоси. Введем функцию на $[0,+\infty)$

$$
G[f](t)=\inf \left\{\varphi: \varphi=\sqrt{a^{2}+b^{2} t^{2}}, \varphi \geqslant f(t)\right\}
$$

Легко видеть, что функция $G[f](t)$ является аналогом вогнутой огибающей функции $f$, построенной с помощью гипербол вместо линейных функций.

Заметим, что если $f(t)$ является инфимумом гипербол, то $G[f]=f$. В частности, для любого $K_{2}(t, \mathbf{x})$ справедливо $G\left[K_{2}\right]=K_{2}$.

TEOpema $2 . K_{2}(t, \mathbf{x})=G\left(\max _{1 \leqslant k \leqslant n} f_{k}\right)(t)$. 
ДокАЗАтЕЛЬСтво. При определении функции $G\left(\max _{1 \leqslant k \leqslant n} f_{k}\right)(t)$ достаточно рассматривать только те гиперболы $\varphi=\sqrt{a^{2}+b^{2} t^{2}}$, которые касаются

$$
f(t)=\max _{1 \leqslant k \leqslant n} f_{k}(t) .
$$

Тогда по лемме 3 существуют векторы $\mathbf{y}_{1}, \mathbf{y}_{2}, \mathbf{y}_{1}+\mathbf{y}_{2}=\mathbf{x}$, такие, что $\left\|\mathbf{y}_{1}\right\|_{1}=a$, $\left\|\mathbf{y}_{2}\right\|_{2}=b$. Поэтому

$$
K_{2}(t)=\inf _{\mathbf{x}=\mathbf{x}_{1}+\mathbf{x}_{2}} \sqrt{\left\|\mathbf{x}_{1}\right\|_{1}^{2}+\left\|\mathbf{x}_{2}\right\|_{2}^{2} t^{2}} \leqslant \sqrt{\left\|\mathbf{y}_{1}\right\|_{1}^{2}+\left\|\mathbf{y}_{2}\right\|_{2}^{2} t^{2}}=\varphi(t)
$$

для любой гиперболы $\varphi$, касающейся $f$. Следовательно, $K_{2}(t) \leqslant G\left(\max _{1 \leqslant k \leqslant n} f_{k}\right)(t)$.

Проверим, что $K_{2}(t) \geqslant G\left(\max _{1 \leqslant k \leqslant n} f_{k}\right)(t)$. Из (4) имеем $K_{2}(t) \geqslant \max _{1 \leqslant k \leqslant n} f_{k}(t)$, следовательно, $K_{2}(t)=G\left[K_{2}\right](t) \geqslant G\left(\max _{1 \leqslant k \leqslant n} f_{k}\right)(t)$. Теорема доказана.

Функции вида $f_{k}(t)$ - это возрастающие вогнутые ограниченные функции. При взятии максимума нескольких таких функций на графике появляются изломы. Эти изломы сглаживаются гиперболами $\varphi=\sqrt{a^{2}+b^{2} t^{2}}$, причем сглаживающая гипербола единственна для каждого излома в силу того, что различные гиперболы могут пересечься лишь в одной точке на $(0,+\infty)$. Таким образом, график $K_{2}(t)$ представляет из себя чередование функций $f_{k}(t)$ и гипербол $\varphi=\sqrt{a^{2}+b^{2} t^{2}}$.

3. Частный случай в бесконечномерном пространстве. Пусть $\alpha=\left\{\alpha_{i}\right\}-$ последовательность положительных чисел, $\ell_{\infty}-$ пространство всех ограниченных последовательностей с нормой $\|\mathbf{x}\|=\sup _{i}\left(\left|x^{i}\right|\right)$. Обозначим через $\ell_{\infty}(\alpha)$ пространство последовательностей $\left\{x^{i}\right\}_{i=1}^{\infty}$ таких, что $\left\{\alpha_{i} x^{i}\right\}$ - ограниченная последовательность, и норма $\|\mathbf{x}\|=\sup _{i}\left(\left|\alpha_{i} x^{i}\right|\right)$.

Рассмотрим пару $\left\{\ell_{\infty}(\alpha), \ell_{\infty}\right\}$, т.е. пусть

$$
\|\mathbf{x}\|_{1}=\sup \left(\alpha_{1}\left|x^{1}\right|, \alpha_{2}\left|x^{2}\right|, \ldots\right), \quad\|\mathbf{x}\|_{2}=\sup \left(\left|x^{1}\right|,\left|x^{2}\right|, \ldots\right) .
$$

ЛЕмма 4. Для пары $\left\{\ell_{\infty}(\alpha), \ell_{\infty}\right\}$ выполняется

$$
K_{2}\left(t,\left\{x^{i}\right\}_{i=1}^{\infty}\right)=\lim _{n \rightarrow \infty} K_{2}\left(t,\left\{x^{i}\right\}_{i=1}^{n}\right),
$$

где $K_{2}\left(t,\left\{x^{i}\right\}_{i=1}^{n}\right)$ - это функиия $K_{2}(t)$, построенная по нормам

$$
\begin{aligned}
& \|\mathbf{x}\|_{1}=\max \left(\alpha_{1}\left|x^{1}\right|, \alpha_{2}\left|x^{2}\right|, \ldots, \alpha_{n}\left|x^{n}\right|\right), \\
& \|\mathbf{x}\|_{2}=\max \left(\left|x^{1}\right|,\left|x^{2}\right|, \ldots,\left|x^{n}\right|\right) .
\end{aligned}
$$

ДокАЗАТЕЛьство. Непосредственно из определения норм вытекает, что

$$
\left\|\left\{x^{i}\right\}_{i=1}^{\infty}\right\|_{1} \geqslant\left\|\left\{x^{i}\right\}_{i=1}^{n}\right\|_{1} \geqslant\left\|\left\{x^{i}\right\}_{i=1}^{n-1}\right\|_{1} .
$$

Обозначив $\mathbf{x}=\left\{x^{i}\right\}_{i=1}^{\infty}, \mathbf{x}^{n}=\left\{x^{i}\right\}_{i=1}^{n}$, из этих неравенств получаем для разбиения $\mathbf{x}=\mathbf{x}_{1}+\mathbf{x}_{2}$

$$
\sqrt{\left\|\mathbf{x}_{1}^{n-1}\right\|_{1}^{2}+\left\|\mathbf{x}_{2}^{n-1}\right\|_{2}^{2} t_{0}^{2}} \leqslant \sqrt{\left\|\mathbf{x}_{1}^{n}\right\|_{1}^{2}+\left\|\mathbf{x}_{2}^{n}\right\|_{2}^{2} t_{0}^{2}} \leqslant \sqrt{\left\|\mathbf{x}_{1}\right\|_{1}^{2}+\left\|\mathbf{x}_{2}\right\|_{2}^{2} t_{0}^{2}} .
$$


Отсюда следует, что $K_{2}(t, \mathbf{x}) \geqslant K_{2}\left(t, \mathbf{x}^{n}\right) \geqslant K_{2}\left(t, \mathbf{x}^{n-1}\right)$. С другой стороны, применяя лемму о сопряженной форме и подставляя $f(\mathbf{x})=\sum_{i=1}^{\infty} \beta_{i} x^{i},\|f\|_{1}=\sum_{i=1}^{\infty} \alpha_{i}^{-1}\left|\beta_{i}\right|$, $\|f\|_{2}=\sum_{i=1}^{\infty}\left|\beta_{i}\right|($ см. $[1,2.7 .1])$, получим

$$
K_{2}=\sup _{\beta} \frac{\left|\sum_{i=1}^{\infty} \beta_{i} x^{i}\right|}{\left(\left(\sum_{i=1}^{\infty} \alpha_{i}^{-1}\left|\beta_{i}\right|\right)^{2}+\left(\frac{1}{t} \sum_{i=1}^{\infty}\left|\beta_{i}\right|\right)^{2}\right)^{1 / 2}} .
$$

Обозначим функцию, стоящую под знаком sup, через $\varkappa(t, \beta)$ и соответственно $\varkappa\left(t, \beta^{n}\right)$ будет та же функция, но суммирование не до бесконечности, а до $n$. Тогда по определению супремума существует такое $\beta_{0}$, что $K_{2}\left(t_{0}, \mathbf{x}\right)-\varkappa\left(t_{0}, \beta_{0}\right)<\varepsilon$. С другой стороны, сушествует такое $n$, что $\left|\varkappa\left(t_{0}, \beta_{0}^{n}\right)-\varkappa\left(t_{0}, \beta_{0}\right)\right|<\varepsilon$. Таким образом, получим $\left|K_{2}\left(t_{0}, \mathbf{x}\right)-\varkappa\left(t_{0}, \beta_{0}^{n}\right)\right|<2 \varepsilon$. Так как

$$
K_{2}\left(t_{0}, \mathbf{x}\right) \geqslant K_{2}\left(t_{0}, \mathbf{x}^{n}\right)=\sup _{\beta^{n}} \varkappa\left(t_{0}, \beta^{n}\right) \geqslant \varkappa\left(t_{0}, \beta_{0}^{n}\right)
$$

то так же получим $\left|K_{2}\left(t_{0}, \mathbf{x}\right)-K_{2}\left(t_{0}, \mathbf{x}^{n}\right)\right|<2 \varepsilon$. Лемма доказана.

Teopema 3. $K_{2}\left(t,\left\{x^{i}\right\}_{i=1}^{\infty}\right)=G\left(\sup _{1 \leqslant k<\infty} f_{k}\right)(t)$.

ДокАЗАТЕЛЬСТво. Заметим, что

$$
K_{2}\left(t,\left\{x^{i}\right\}_{i=1}^{n}\right)=G\left(\max _{1 \leqslant k \leqslant n} f_{k}\right)(t) \leqslant G\left(\sup _{1 \leqslant k<\infty} f_{k}\right)(t)
$$

Переходя к пределу при $n \rightarrow \infty$ получим

$$
K_{2}\left(t,\left\{x^{i}\right\}_{i=1}^{\infty}\right) \leqslant G\left(\sup _{1 \leqslant k<\infty} f_{k}\right)(t)
$$

С другой стороны,

$$
K_{2}\left(t,\left\{x^{i}\right\}_{i=1}^{\infty}\right) \geqslant K_{2}\left(t,\left\{x^{i}\right\}_{i=1}^{n}\right) \geqslant \max _{1 \leqslant k \leqslant n} f_{k}(t)
$$

Поэтому

$$
K_{2}\left(t,\left\{x^{i}\right\}_{i=1}^{\infty}\right) \geqslant \sup _{1 \leqslant k<\infty} f_{k}(t),
$$

следовательно,

$$
K_{2}\left(t,\left\{x^{i}\right\}_{i=1}^{\infty}\right) \geqslant G\left(\sup _{1 \leqslant k<\infty} f_{k}\right)(t) .
$$

Теорема доказана. 


\section{4. Функции, имеющие представление в виде $K_{2}$-функционала.}

ТЕорема 4. Любая непрерывная функция, которая может быть представлена как $f(t)=\sup _{\xi, \alpha} \xi \sqrt{\alpha^{2} t^{2} /\left(\alpha^{2}+t^{2}\right)}=\inf _{c, d} \sqrt{c^{2}+d^{2} t^{2}}$ для некоторого множества $A$ положительных параметров $\alpha, \xi, c, d$, может быть получена в качестве $K_{2}$-функиионала от некоторого вектора в некоторой банаховой паре.

ДокАЗАтельство. Так как $\mathbb{R}^{1}$ сепарабельно, рассмотрим множество $B=\left\{b_{i}\right\}$ счетное всюду плотное на $\mathbb{R}^{1}$. Для каждого $b_{i}$ найдется счетное подмножество $A_{i}$ множества параметров $A$ такое, что

$$
f\left(b_{i}\right)=\sup _{\xi, \alpha \in A_{i}} \xi \sqrt{\frac{\alpha^{2} b_{i}^{2}}{\alpha^{2}+b_{i}^{2}}}
$$

Множество $A^{\prime}=\bigcup_{i} A_{i}$ будет счетньм. Перенумеруем все $\xi, \alpha \in A^{\prime}$. Рассмотрим пару $\left\{\ell_{\infty}(\alpha), \ell_{\infty}\right\}$, где $\alpha=\left\{\alpha_{i}\right\}_{i=1}^{\infty}$ и вектор $\mathbf{x}=\left\{\xi_{i}\right\}_{i=1}^{\infty}$. Тогда получим

$$
\begin{aligned}
K_{2}(t)=G\left(\sup _{\xi, \alpha \in A^{\prime}} \xi_{i} \sqrt{\frac{\alpha_{i}^{2} t^{2}}{\alpha_{i}^{2}+t^{2}}}\right) & =G\left(\sup _{\xi, \alpha \in A} \xi \sqrt{\frac{\alpha^{2} t^{2}}{\alpha^{2}+t^{2}}}\right) \\
& =G[f](t)=f(t)
\end{aligned}
$$

на множестве $B$, а, следовательно, и на всей полуоси, в силу непрерьвности $f(t)$. Теорема доказана.

Из теоремы 4 непосредственно вытекает следующая теорема о возможности сведения общего случая функции $K_{2}(t)$ к рассмотренному вьше частному.

Tеорема 5. Пусть $X_{1}, X_{2}$ - банахова пара, тогда для любого $\mathbf{x} \in X_{1}+X_{2}$ найдутся последовательности $\alpha=\left\{\alpha_{i}\right\}$ и $\mathbf{x}^{\prime}=\left\{\xi^{i^{\prime}}\right\}$, ито

$$
K_{2}\left(t, \mathbf{x}, X_{1}, X_{2}\right)=K_{2}\left(t, \mathbf{x}^{\prime}, \ell_{\infty}(\alpha), \ell_{\infty}\right) .
$$

ДокАЗАТЕЛЬСТво. По определению

$$
K_{2}(t, \mathbf{x})=\inf _{\mathbf{x}=\mathbf{x}_{1}+\mathbf{x}_{2}} \sqrt{\left\|\mathbf{x}_{1}\right\|_{1}^{2}+t^{2}\left\|\mathbf{x}_{2}\right\|_{2}^{2}}
$$

т.e.

$$
K_{2}(t)=\inf _{c, d} \sqrt{c^{2}+d^{2} t^{2}}
$$

для некоторого множества параметров $c, d$. С другой стороны, по лемме 1

$$
K_{2}(t, \mathbf{x})=\sup _{f \neq 0} \frac{|f(\mathbf{x})|}{\left(\|f\|_{1}^{2}+t^{-2}\|f\|_{2}^{2}\right)^{1 / 2}}=\sup _{\xi, \alpha} \xi \sqrt{\frac{\alpha^{2} t^{2}}{\alpha^{2}+t^{2}}},
$$

где $\xi=|f(\mathbf{x})| /\|f\|_{2}, \alpha=\|f\|_{2} /\|f\|_{1}$. Значит, функция $K_{2}(t, \mathbf{x})$ удовлетворяет условиям предыдущей теоремы. Теорема доказана.

Определим функцию $L[f](t)$ как вогнутую огибающую положительной функции $f$ :

$$
L[f](t)=\inf \{\varphi=a+b t: \varphi \geqslant f(t)\} .
$$


Теорема 6. Функиия $F(t)=L[f](t)$, әде

$$
f(t)=\sup _{\xi, \alpha} \xi \sqrt{\frac{\alpha^{2} t^{2}}{\alpha^{2}+t^{2}}}
$$

для некоторого множества положительных параметров $\xi, \alpha$, может быть получена в качестве $K_{2}$-функционала от некоторого вектора в некоторой банаховой nape.

ДокАЗАТЕЛЬСТво. Как вогнутая функция $F(t)$ может быть представлена в качестве инфимума гипербол $\varphi(t)=\sqrt{c^{2}+d^{2} t^{2}}$, и, если мы докажем, что

$$
F(t)=\sup _{\zeta, \beta} \zeta \sqrt{\frac{\beta^{2} t^{2}}{\beta^{2}+t^{2}}}
$$

для некоторых $\zeta, \beta$, то получим искомьй результат непосредственно из теоремы 4.

Рассмотрим произвольную точку $t_{0}$. Легко видеть, что возможны два варианта: или $f\left(t_{0}\right)=F\left(t_{0}\right)$, или существуют такие $t_{1}, t_{2}, t_{1}<t_{0}<t_{2}$, что на отрезке $\left[t_{1}, t_{2}\right]$ функция $F(t)$ представляет собой линейную функцию, причем $f\left(t_{1}\right)=F\left(t_{1}\right)$ и $f\left(t_{2}\right)=F\left(t_{2}\right)$. В первом случае равенство (6) в точке $t_{0}$ выполняется автоматически. Докажем, что оно справедливо во втором случае.

Нетрудно видеть, что существуют такие $\zeta, \beta$, что функщия

$$
g(t)=\zeta \sqrt{\frac{\beta^{2} t^{2}}{\beta^{2}+t^{2}}}
$$

касается функции $F(t)$ снизу в точке $t_{0}$, следовательно, $g\left(t_{0}\right)>f\left(t_{0}\right)$. С другой стороны, по свойству супремума, найдется такая функция

$$
g_{1}(t)=\xi \sqrt{\frac{\alpha^{2} t^{2}}{\alpha^{2}+t^{2}}},
$$

что $f\left(t_{1}\right) \geqslant g_{1}\left(t_{1}\right)>g\left(t_{1}\right)$. Так как уравнение $g(t)=g_{1}(t)$ может иметь только одно решение на полуоси $(0,+\infty)$ и оно, очевидно, лежит между $t_{1}$ и $t_{0}$, то на промежутке $\left(0, t_{1}\right)$ выполнено неравенство $g(t)<g_{1}(t)$, и, следовательно, неравенство $g(t)<f(t) \leqslant F(t)$. Аналогичными рассуждениями приходим к выводу, что $g(t)<F(t)$ и на промежутке $\left(t_{2},+\infty\right)$. Таким образом, $g(t) \leqslant F(t)$ на всей полуоси $(0,+\infty)$ и в точке $t_{0}$ условие $(6)$ выполнено. Теорема доказана.

\section{СПИСОК ЦИТИРОВАННОЙ ЛИТЕРАТУРЫ}

[1] Берг $\breve{И} .$, Лёфстрем Й. Интерполяционные пространства. Введение. М.: Мир, 1980.

[2] Овчинников В.И. Интерполяционные теоремы, вытекающие из неравенства Гротендика // Функцион. анализ и его прилож. 1976. Т. 10. №4. С. 45-54.

[3] Бурбаки Н. Функции действительного переменного. М.: Наука, 1965. 